\title{
A REMARK ON DYNAMICAL DEGREES OF AUTOMORPHISMS OF HYPERKÄHLER MANIFOLDS
}

\author{
KEIJI OGUISO
}

To the memory of Professor Masayoshi Nagata

\begin{abstract}
We describe all the dynamical degrees of automorphisms of hyperkähler manifolds in terms of the first dynamical degree. We also present two explicit examples of different geometric flavours.
\end{abstract}

\section{INTRODUCTION}

A hyperkähler manifold is a simply connected compact Kähler manifold $X$ such that $H^{0}\left(X, \Omega_{X}^{2}\right)=\mathbb{C} \sigma_{X}$, where $\sigma_{X}$ is an everywhere non-degenerate holomorphic 2 -form on $X$. It is then of even dimension.

The aim of this note is to point out the following simple, explicit behaviour of the dynamical degrees of automorphisms of hyperkähler manifolds (see Section 2 for terminologies).

1.1. Theorem. Let $X$ be a hyperkähler manifold of dimension $2 n$ and $g$ be a holomorphic automorphism of $X$. Let $k$ be an integer such that $0 \leq k \leq 2 n$ and $d_{k}(g)$ be the $k$-th dynamical degree of $g$. Then

$$
d_{2 n-k}(g)=d_{k}(g)=d_{1}(g)^{k}
$$

for $0 \leq k \leq n$. In particular, if $g$ is of positive entropy, then

$$
1=d_{0}(g)<d_{1}(g)<\cdots<d_{n-1}(g)<d_{n}(g)>d_{n+1}(g)>\cdots>d_{2 n}(g)=1,
$$

i.e., the function

$$
\mathbb{Z} \cap[0, n] \ni k \mapsto d_{k}(g) \in \mathbb{R}
$$

is strictly increasing. Moreover, the topological entropy $h(g)$ of $g$ is

$$
h(g)=n \log d_{1}(g) .
$$

This behaviour fits well with the assumption made in DS09 Section 4.4. So, one can now apply the results there for automorphisms of hyperkähler manifolds.

A Salem number is a real algebraic integer $\alpha$ such that $\alpha>1,1 / \alpha$ is a Galois conjugate of $\alpha$ and all other Galois conjugates are of absolute value 1. Salem numbers play important roles in complex dynamics on K3 surfaces, rational surfaces ([Mc02, [Mc07]) and hyperkähler manifolds ( $\mathrm{Og} 07$ and references therein).

1.2. Corollary. The dynamical degrees of automorphisms of hyperkähler manifolds are Salem numbers or 1.

Date: November 3, 2018.

2000 Mathematics Subject Classification. 14J50 (14J28 14J40 37B40 53C26). 
Dynamical degrees are natural, important invariants in complex dynamics of automorphisms, but there are very few examples in dimension $\geq 3$ for which explicit computations are made.

The next aim of this note is to give a few explicit computaions of dynamical degrees of automorphisms of $S^{[n]}$ (Examples 1, 2 in Section 4). Here and hereafter $S^{[n]}$ is the Hilbert scheme of 0-dimensional subschemes of length $n$ on a K3 surface $S$. As it is well known, $S^{[n]}$ is a $2 n$-dimensional hyperkähler manifold ( $\mathrm{Fu} 83$ for $n=2$, Be83. for any $n \geq 2$ ).

Example 1 is elementary and everything is very concrete, but automorphisms under consideration are all induced from automorphisms of $S$. In Example 2, we construct automorphisms of positive entropy of some $S^{[2]}$ that are not induced from any automorphisms of any $S^{\prime}$ such that $\left(S^{\prime}\right)^{[2]} \simeq S^{[2]}$. A similar construction in a more general context was also made by O'Grady OGr05, Section 4 (see especially subsection 4.4). For our explicit computation of the dynamical degrees, the specification made in Lemma 4.5 is convenient.

Theorem 1.1 and Examples 1, 2 will partially answer to the question of Professor Nessim Sibony to me (June 2008):

1.3. Question. Besides automorphisms of complex tori or automorphisms of product type (DS09] Section 4.4), are there any explicit examples of automorphisms $f$ of higher dimensional manifolds $M$ such that the dynamical degrees $d_{k}(f)(k \in$ $[0, \operatorname{dim} M / 2] \cap \mathbb{Z})$ are strictly increasing, i.e., mutually distinct?

In the view of Corollary 1.2 the following question might be of interest:

1.4. Question. Which Salem numbers are realizable as dynamical degrees of automorphisms of hyperkähler manifolds? In particular, is the Lehmer number, i.e., the smallest known Salem number

$$
\lambda_{\text {Lehmer }}=1.17628 \ldots
$$

realizable in this way?

We note that the Lehmer number is realizable as the first dynamical degree of a rational surface automorphism [Mc07].

\section{Dynamical Degrees of AUtomorPhisms - A FEW KNOWN RESUlts}

In this section, we briefly review some basic notions and some known properties of dynamical degrees. Our sources are DS05 Section 2 and Gu05 Section 1. Zh08 Section 2 also provides a very good survey particularly for algebraic geometers. For more details or any history, see these papers and the references therein.

Let $M$ be a compact Kähler manifold of dimension $m$ and $f$ be a biholomorphic automorphism of $M$. Let $k$ be an integer such that $0 \leq k \leq m$. Then, the $k$-th dynamical degree $d_{k}(f)$ of $f$ is defined by

$$
d_{k}(f)=\lim _{\ell \longrightarrow \infty}\left(\int_{M}\left(f^{\ell}\right)^{*} \eta^{k} \wedge \eta^{m-k}\right)^{1 / \ell} .
$$

Here $\eta$ is a Kähler form on $M$. It is well known that $d_{k}(f)$ does not depend on the choice of $\eta$. Moreover,

$$
d_{k}(f)=\rho\left(f^{*} \mid H^{k, k}(M, \mathbb{R})\right)=\rho\left(f^{*} \mid H^{2 k}(M, \mathbb{R})\right) .
$$


Here $\rho(*)$ denotes the spectral radius of the linear map *. Probably, the first important property of $d_{k}(f)$ is the following symmetry:

$$
d_{k}(f)=d_{m-k}\left(f^{-1}\right) .
$$

This follows from the Poincaré duality. As a more interesting property, it is also known that the function

$$
[0, m] \ni k \mapsto \log d_{k}(f) \in \mathbb{R}
$$

is always concave (see eg. Gu05 Proposition 1.2, DS05 Proposition 2.6). From these two properties and $d_{0}(f)=1$, we have the following general behaviour of the dynamical degrees:

2.1. Theorem. There are $p, q \in[0, m] \cap \mathbb{Z}$ such that $p \leq q$ and

$$
1=d_{0}(f)<\cdots<d_{p}(f)=\cdots=d_{q}(f)>\cdots>d_{m}(f)=1 .
$$

The topological entropy $h(f)$ of $f$ is a real number that measures the complexity of orbit space

$$
x, f(x), f^{2}(x), f^{3}(x), \cdots, f^{m}(x), \cdots .
$$

Original definition of $h(f)$ is purely topological (Gr03, Section 1), and the action on the cohomology ring never appears in the definition. However, for an automorphism $f$ of a compact Kähler manifold $X$, it turns out that

$$
h(f)=\log d_{p}(f)=\max \left\{\log d_{k}(f) \mid k \in[0, m] \cap \mathbb{Z}\right\},
$$

by the fundamental theorem due to Gromov and Yomdin. In this note, we only need this fact. Note then that $h(f) \geq 0$, and $h(f)>0$ if and only if $d_{1}(f)>1$ and that the function $d_{k}(f)$ is constantly 1 when $h(f)=0$.

So, our new Theorem 1.1 says that only two extreme cases in Theorem 2.1 can happen for automorphisms of hyperkähler manifolds. That is, according to $h(g)=0$ or $h(g)>0$, either $p=0$ and $q=\operatorname{dim} X$ or $p=q=n=\operatorname{dim} X / 2$.

\section{Proof of Theorem 1.1 and Corollary 1.2}

In this section, we shall prove Theorem 1.1 and Corollary 1.2 The proof is extremely simple.

Let $k \in[0, n] \cap \mathbb{Z}$. Consider the natural map

$$
m_{k}: \operatorname{Sym}^{k} H^{2}(X, \mathbb{R}) \longrightarrow H^{2 k}(X, \mathbb{R}) \text {. }
$$

$m_{k}$ is given by the multiplication of the cohomology $\operatorname{ring} H^{*}(X, \mathbb{R})$. In particular, $m_{k}$ is compatible with the action of Aut $X$. Moreover, by a result of Verbitsky Ve96] (see also [Bo96] together with B078, [CY03] Part III, Proposition 24.1), the map $m_{k}$ is injective for $k$ in the range above. Thus $d_{k}(g) \geq d_{1}(g)^{k}$, i.e.,

$$
\log d_{k}(g) \geq k \log d_{1}(g) .
$$

On the other hand, as $k \mapsto \log d_{k}(g)$ is a concave function with $\log d_{0}(g)=0$, we have that

$$
\log d_{k}(g) \leq k \log d_{1}(g) .
$$

Thus, $\log d_{k}(g)=k \log d_{1}(g)$, and hence $d_{k}(g)=d_{1}(g)^{k}$ for each $k \in[0, n] \cap \mathbb{Z}$.

On the other hand, by $\mathrm{Og} 07$ Section 2.2, the characteristic polynomial of $g^{*} \mid H^{2}(X, \mathbb{Z})$ has at most one Salem polynomial factor, i.e., the minimal polynomial of a Salem number over $\mathbb{Z}$, and the other factors are all cyclotomic polynomials. 
Thus, the set of eigenvalues of $g$ and $g^{-1}$ are the same. We should note that this is a very special property of an automorphism of hyperkähler manifolds. In particular,

$$
d_{1}(g)=d_{1}\left(g^{-1}\right) .
$$

Hence,

$$
d_{2 n-k}(g)=d_{k}\left(g^{-1}\right)=d_{1}\left(g^{-1}\right)^{k}=d_{1}(g)^{k} .
$$

This completes the proof of Theorem 1.1

Let us show Corollary 1.2 The result is clear if $h(g)=0$. Assume that $h(g)>0$. Then, again by $\mathrm{Og} 07$ Section 2.2, the characteristic polynomial of $g^{*} \mid H^{2}(X, \mathbb{Z})$ has a Salem polynomial factor. Thus, $d_{1}(g)$ is a Salem number. Let $\gamma$ be a Galois conjugate of $d_{1}(g)^{k}$. Then, $\gamma$ is of the form $\beta^{k}$ for some Galois conjugate $\beta$ of $d_{1}(g)$ and vice versa. This implies the result.

3.1. Remark. Let $X$ be a hyperkähler 4 -fold which is deformation equivalent to the Hilbert scheme of a K3 surface, say, $S^{[2]}$. Then $b_{2}(X)=b_{2}\left(S^{[2]}\right)=23$ and $b_{4}(X)=b_{4}\left(S^{[2]}\right)=276$. (This is well known and is indeed easy to see from the explicit description of $S^{[2]}$.) Thus, the injective map $m_{2}$ is actually an isomorphism for dimension reason and we have that $H^{4}(X, \mathbb{R})=\operatorname{Sym}^{2} H^{2}(X, \mathbb{R})$. Thus, if $g \in$ Aut $X$ is of positive entropy, then the dynamical degree $d_{k}(g)$ is an eigenvalue of $g^{*} \mid H^{2 k}(X, \mathbf{R})$ of multiplicity one for each $k$. It would be interesting to see to what extent this multiplicity one property of dynamical degrees holds for automorphisms of hyperkähler manifolds of positive entropy.

3.2. Remark. Recall that the Hilbert scheme $F(V)$ of lines on a smooth cubic 4-fold $V$ is deformation equivalent to $S^{[2]}$ BD85]. In [Am07, Amerik computed the dynamical degrees of a natural, very interesting self rational map of degree 16 on $F(V)$. They are

$$
d_{0}=1, d_{1}=7, d_{2}=31, d_{3}=28, d_{4}=16 .
$$

It is remarkable that the behaviour is quite different from the case of automorphisms in Theorem 1.1. This difference is pointed out to us by Professor Frédéric Campana.

\section{A feW EXPlicit EXAMPLes}

Let $S$ be a K3 surface and $S^{[n]}$ be the Hilbert scheme of $n \geq 2$ points on $S$. In this section, we shall compute the dynamical degrees of some automorphisms of $S^{[n]}$ in two specific situations (Examples 1,2).

4.1. Lemma. Let $g$ be an automorphism of $S$ and $g_{n}$ be the automorphism of $S^{[n]}$ induced from $g$. Then $d_{1}\left(g_{n}\right)=d_{1}(g)$, and $d_{2 n-k}\left(g_{n}\right)=d_{k}\left(g_{n}\right)=d_{1}(g)^{k}$ for $k \in[0, n] \cap \mathbb{Z}$. In particular, $h\left(g_{n}\right)=n \log d_{1}(g)$.

Proof. Let $E$ be the exceptional divisor of the Hilbert-Chow morphism

$$
S^{[n]} \longrightarrow S^{(n)}=S^{n} / \Sigma_{n} .
$$

Then, by [Be83] (see also [CY03] Part III, Section 23), $H^{2}\left(S^{[n]}, \mathbb{Z}\right)$ carries a natural integral-valued symmetric bilinear form, called the Beauville-Bogomolov-Fujiki's form. Via the natural isomorphism $H^{2}(S, \mathbb{Z}) \simeq H^{2}\left(S^{n}, \mathbb{Z}\right)^{\Sigma_{n}}$, we can embed $H^{2}(S, \mathbb{Z})$ into $H^{2}\left(S^{[n]}, \mathbb{Z}\right)$. Be83] shows that this embedding gives a natural Hodge isometry

$$
H^{2}\left(S^{[n]}, \mathbb{Z}\right) \simeq H^{2}(S, \mathbb{Z}) \oplus \mathbb{Z}[e] .
$$


Here $e:=E / 2 \in H^{2}\left(S^{[n]}, \mathbb{Z}\right)$ and $\left(e^{2}\right)=-2 n+2$. Since $g_{n}$ is induced from $g$, under the isometry above, we have that $g_{n}^{*}(e)=e, g_{n}^{*}\left(H^{2}(S, \mathbb{Z})\right)=H^{2}(S, \mathbb{Z})$ and $g_{n}^{*}\left|H^{2}(S, \mathbb{Z})=g^{*}\right| H^{2}(S, \mathbb{Z})$. Thus, $d_{1}\left(g_{n}\right)=d_{1}(g)$. This together with Theorem 1.1 implies the result.

Example 1. Let $E$ be an elliptic curve. Put $A=E \times E$. Let $S=\operatorname{Km}(A)$ be the Kummer K3 surface associated with $A$, i.e., the minimal resolution of the quotient surface $A /\langle-1\rangle$. Let

$$
M:=\left(\begin{array}{ll}
a & b \\
c & d
\end{array}\right) \in \mathrm{SL}(2, \mathbb{Z}) .
$$

Then $M$ defines an automorphism $f_{M}$ of $A$ by

$$
A \ni(x, y) \mapsto(a x+b y, c x+d y) \in A .
$$

Since $f_{M}$ commutes with the inversion -1 of $A$, it descends to an automorphism $g_{M}$ of $S$, and therefore, induces an automorphism $g_{M, n}$ on $S^{[n]}$. Recall that

$$
H^{2}(S, \mathbb{Q}) \simeq H^{2}(A, \mathbb{Q}) \oplus \oplus_{i=1}^{16} \mathbb{Q}\left[e_{i}\right],
$$

where $e_{i}(1 \leq i \leq 16)$ are the exceptional divisors of $S \longrightarrow A /\langle-1\rangle$. This isomorphism is compatible with the actions of $f_{M}$ and $g_{M}$. In particular, the subspaces $\oplus_{i=1}^{16} \mathbb{Q}\left[e_{i}\right]$ is stable under the action. Moreover, the cup product is negative definite on $\oplus_{i=1}^{16} \mathbb{Q}\left[e_{i}\right]$. Thus, $d_{1}\left(g_{M}\right)=d_{1}\left(f_{M}\right)$. Hence $d_{1}\left(g_{M, n}\right)=d_{1}\left(f_{M}\right)$ and $h\left(g_{M, n}\right)=n \log d_{1}\left(f_{M}\right)$ by Lemma 4.1

4.2. Lemma. Let $t=a+d$,

$$
\alpha=\frac{t+\sqrt{t^{2}-4}}{2}, \beta=\frac{t-\sqrt{t^{2}-4}}{2} .
$$

$\alpha$ and $\beta$ are the eigenvalues of the matrix $M$ counted with multiplicities. Then

$$
\begin{gathered}
d_{1}\left(f_{M}\right)=1 \text { if }|t| \leq 2, \\
d_{1}\left(f_{M}\right)=\alpha^{2}>1 \text { if } t>2, \\
d_{1}\left(f_{M}\right)=\beta^{2}>1 \text { if } t<-2 .
\end{gathered}
$$

Proof. $d_{1}\left(f_{M}\right)$ is the spectral radius of $f_{M}^{*} \mid H^{1,1}(A, \mathbb{R})$. Since $A$ is a 2-dimensional complex torus, we have the following natural isomorphism:

$$
H^{1,1}(A, \mathbb{R}) \otimes \mathbb{C}=H^{1,1}(A, \mathbb{C}) \simeq H^{0}\left(A, \Omega_{A}^{1}\right) \otimes \overline{H^{0}\left(A, \Omega_{A}^{1}\right)} .
$$

Thus, the eigenvalues of $f_{M}^{*} \mid H^{1,1}(A, \mathbb{R})$ are $\alpha \bar{\alpha}, \alpha \bar{\beta}, \beta \bar{\alpha}, \beta \bar{\beta}$, counted with multiplicities. This implies the result.

Hence, according to $|t| \leq 2, t>2, t<-2$, we have

$$
\begin{gathered}
d_{k}\left(g_{M, n}\right)=1(\forall k \in[0,2 n] \cap \mathbb{Z}), \\
d_{2 n-k}\left(g_{M, n}\right)=d_{k}\left(g_{M, n}\right)=\left(\frac{t+\sqrt{t^{2}-4}}{2}\right)^{2 k}(\forall k \in[0, n] \cap \mathbb{Z}), \\
d_{2 n-k}\left(g_{M, n}\right)=d_{k}\left(g_{M, n}\right)=\left(\frac{t-\sqrt{t^{2}-4}}{2}\right)^{2 k}(\forall k \in[0, n] \cap \mathbb{Z}) .
\end{gathered}
$$

For the entropy, $h\left(g_{M, n}\right)=0$ in the first case, and $h\left(g_{M, n}\right)>0$ in the last two cases.

Example 2. In this example, we present automorphisms of positive entropy of some $S^{[2]}$ that are not induced from automorphisms of any $S^{\prime}$ such that $\left(S^{\prime}\right)^{[2]} \simeq S^{[2]}$. 
Note that there is a pair of K3 surfaces $X, Y$ such that $X \not Y$ but $X^{[2]} \simeq Y^{[2]}$ (Yo01 Example 7.2).

Recall the natural identification (made in the proof of Lemma 4.1)

$$
H^{2}\left(S^{[2]}, \mathbb{Z}\right)=H^{2}(S, \mathbb{Z}) \oplus \mathbb{Z}[e] .
$$

Under this identification, we have

$$
N S\left(S^{[2]}\right)=N S(S) \oplus \mathbb{Z}[e] .
$$

The involution $\iota$ in the next Lemma is constructed by Beauville [Be82]:

4.3. Lemma. Let $S \subset \mathbb{P}^{3}$ be a quartic K3 surface. Then the line $\overline{P_{1} P_{2}}$ joining two general points $P_{1}, P_{2}$ of $S$ meets $S$ in two other points, say, $P_{3}, P_{4}$. The correspondence $\left\{P_{1}, P_{2}\right\} \mapsto\left\{P_{3}, P_{4}\right\}$ defines the birational involution

$$
\iota: S^{[2]-\rightarrow S^{[2]}} \text {. }
$$

For this involution, one has:

(1) $\iota$ is a biholomorphic involution if and only if $S$ contains no line.

(2) Under the assumption (1), $\iota^{*} H=3 H-4 e$ and $\iota^{*} e=2 H-3 e$. Here $H$ is the element of $N S\left(S^{[2]}\right)$ corresponding to the hyperplane class of $S$.

(3) Under the assumption (1), $H^{2}\left(S^{[2]}, \mathbb{Z}\right)^{\iota^{*}}=\mathbb{Z}\langle H-e\rangle$.

Proof. (1) is proved by $\mathrm{Be} 82$ Proposition 11. When $N S(S)=\mathbb{Z} H$, i.e., the generic case, (2) is proved by [HT01. Subsection 5.2. For arbitrary $S$ with no line, we shall prove by reducing to the generic case via deformation.

Let us consider a general 1-dimensional small deformation of $S$ inside $\mathbb{P}^{3}$ :

$$
\mathbb{P}^{3} \times \Delta \supset \mathcal{S} \longrightarrow \Delta, \mathcal{S}_{0}=S \text {. }
$$

Let $\mathcal{H}$ be the relative hyperplane class of $\mathcal{S}$ over $\Delta$. Then $N S\left(\mathcal{S}_{t}\right)=\mathbb{Z}\left\langle\mathcal{H}_{t}\right\rangle$ for generic $t \in \Delta$. Put

$$
\Delta_{2}=\left\{s \in \Delta \mid \operatorname{rank} N S\left(\mathcal{S}_{s}\right) \geq 2\right\} .
$$

This set $\Delta_{2}$ is topologically dense in $\Delta$ but it consists of at most countably many points $([\mathrm{Og} 03$ Theorem 1.1). As $\mathbb{R}$ is an uncountable set, for generic $c \in \mathbb{R}$, the real analytic line

$$
\gamma_{c}=\{z \in \Delta \mid \operatorname{Im} z=c \cdot \operatorname{Re} z\} \subset \Delta
$$

then satisfies

$$
0 \in \gamma_{c} \text { and } \gamma_{c} \cap\left(\Delta_{2} \backslash\{0\}\right)=\emptyset .
$$

By construction, $N S\left(\mathcal{S}_{t}\right)=\mathbb{Z}\left\langle\mathcal{H}_{t}\right\rangle$ for $t \in \gamma_{c} \backslash\{0\}$. By taking the relative Hilbert scheme over $\Delta$ and then restricting it to $\gamma_{c}$, we have an analytic family

$$
f: \mathcal{M} \longrightarrow \gamma_{c}
$$

such that $\mathcal{M}_{t}=\mathcal{S}_{t}^{[2]}$ for all $t \in \gamma_{c}$. Let $\mathcal{E}$ be the exceptional divisor of the relative Hilbert-Chow morphism over $\gamma_{c}$. Then, $N S\left(\mathcal{M}_{t}\right)=\mathbb{Z}\left\langle\mathcal{H}_{t}, e_{t}\right\rangle$ for all $t \in \gamma_{c} \backslash\{0\}$ and $N S\left(S^{[2]}\right) \supset \mathbb{Z}\langle H, e\rangle$ for $t=0$. Here $e_{t}=\mathcal{E}_{t} / 2, H=\mathcal{H}_{0}$ and $e=e_{0}$. In particular, $\mathbb{Z}\left\langle\mathcal{H}_{t}, e_{t}\right\rangle\left(t \in \gamma_{c}\right)$ forms a constant subsystem $\Lambda$ of the constant system $R^{2} f_{*} \mathbb{Z}_{\mathcal{M}}$.

As $\mathcal{S}_{t}\left(t \in \gamma_{c}\right)$ contains no $\mathbb{P}^{1}$, the involution $\iota$ extends to a (not only birational but also) biholomorphic involution $I$ of $\mathcal{M}$ over $\gamma_{c}$ such that $I_{0}=\iota$. Under the natural action of $I$ on $R^{2} f_{*} \mathbb{Z}_{\mathcal{M}}$, the constant subsystem $\Lambda$ is stable. This is because the assertion (2) is true for $\mathcal{M}_{t}\left(t \in \gamma_{c} \backslash\{0\}\right)$ by [HT01. Subsection 5.2. Hence, by specializing the result to $t=0$, we have the assertion (2) also for our $S^{[2]}$. 
Let us show the assertion (3). Let $Z \in S^{[2]}$ be a general fixed point of $\iota$. Then $Z=P+Q$, where $P \neq Q$ are the tangent points of a double tangent line $\ell$ of a general hyperplane $H \cap S$ of $S$ (canonical curve of genus 3 ). Let $\left(x_{P}, y_{P}\right)$ be a local coordinate of $P$ in $S$ such that $x_{P}$ is the local coordinate of $H \cap S$ at $P$ and $y_{P}$ is the local coordinate of the one parameter small deformation of the double tangent points at $P$. We take a local coordinate $\left(x_{Q}, y_{Q}\right)$ of $Q$ in $S$ similarly. Consider the local holomorphic 2-form

$$
\tilde{\sigma}=d x_{P} \wedge d y_{P}+d x_{Q} \wedge d y_{Q}
$$

on $S \times S$ at $(P, Q)$. This $\tilde{\sigma}$ is a non-zero multiple of $p_{1}^{*} \sigma_{S}+p_{2}^{*} \sigma_{S}$ at $(P, Q)$, possibly after rescalling of $\left(x_{Q}, y_{Q}\right)$. Here $p_{i}: S \times S \longrightarrow S$ is the projection to the $i$-th factor. Thus $\tilde{\sigma}$ descends to the local holomorphic 2 -form, say $\sigma$, of $S^{[2]}$ at $P+Q$. By definition of $\iota$, we have $\iota^{*} \sigma=-\sigma$. On the other hand, the global holomorphic 2 -form $p_{1}^{*} \sigma_{S}+p_{2}^{*} \sigma_{S}$ descends to the global holomorphic 2 -form $\sigma_{S^{[2]}}$ on $S^{[2]}$. Hence

$$
\iota^{*} \sigma_{S^{[2]}}=-\sigma_{S^{[2]}}
$$

at $P+Q$, whence, on $S^{[2]}$ as well. The same is clearly true for the involution $I_{t}$ on $\mathcal{M}_{t}\left(t \in \gamma_{c}\right)$. Thus

$$
I_{t}^{*} \sigma_{\mathcal{M}_{t}}=-\sigma_{\mathcal{M}_{t}} \text { and } I_{t}^{*} \mid T\left(\mathcal{M}_{t}\right)=-1 .
$$

Here $T\left(\mathcal{M}_{t}\right)$ is the transcendental lattice of $\mathcal{M}_{t}$, i.e., the minimal primitive submodule of $H^{2}\left(\mathcal{M}_{t}, \mathbb{Z}\right)$ such that $\sigma_{\mathcal{M}_{t}} \in T\left(\mathcal{M}_{t}\right)_{\mathbb{C}}$. The second equality follows from the first one via the minimality of $T\left(\mathcal{M}_{t}\right)$. On the other hand,

$$
N S\left(\mathcal{M}_{t}\right)^{I_{t}^{*}}=\mathbb{Z}\left\langle\mathcal{H}_{t}-e_{t}\right\rangle
$$

for all $t \in \gamma_{c} \backslash\{0\}$, by $N S\left(\mathcal{M}_{t}\right)=\mathbb{Z}\left\langle\mathcal{H}_{t}, e_{t}\right\rangle$ and by the explicit form of $I_{t}^{*} \mid N S\left(\mathcal{M}_{t}\right)$ in (2). As $N S\left(\mathcal{M}_{t}\right) \oplus T\left(\mathcal{M}_{t}\right)$ is of finite index submodule of $H^{2}\left(\mathcal{M}_{t}, \mathbb{Z}\right)$, we have then that

$$
H^{2}\left(\mathcal{M}_{t}, \mathbb{Z}\right)^{I_{t}^{*}}=\mathbb{Z}\left\langle\mathcal{H}_{t}-e_{t}\right\rangle
$$

for all $t \in \gamma_{c} \backslash\{0\}$. As $I$ acts on the constant system $R^{2} f_{*} \mathbb{Z}_{\mathcal{M}}$, by specializing to $t=0$, we have

$$
H^{2}\left(S^{[2]}, \mathbb{Z}\right)^{\iota^{*}}=\mathbb{Z}\langle H-e\rangle
$$

as well. This implies the assertion (3).

4.4. Lemma. Let $N=\mathbb{Z}\left\langle h_{1}, h_{2}\right\rangle$ be a lattice whose bilinear form is

$$
\left(\left(h_{i}, h_{j}\right)\right)=\left(\begin{array}{ll}
4 & 8 \\
8 & 4
\end{array}\right) .
$$

Then, there are a projective K3 surface $S$ and an isometry $\varphi: N \simeq N S(S)$ such that both $H_{i}:=\varphi\left(h_{i}\right)(i=1,2)$ are very ample on $S$. This $S$ contains no $\mathbb{P}^{1}$.

Proof. By the explicit form, $N$ is an even lattice of signature $(1,1)$. Thus, by Mo84 Corollary 2.9, there is a projective K3 surface $S$ such that $N \simeq N S(S)$, say by $\varphi$. Let $A(S) \subset N S(S)_{\mathbb{R}}$ be the ample cone of $S$. Again by the explicit form, the lattice $N$ represents neither 0 nor -2 . Thus, $S$ has no $\mathbb{P}^{1}$, and therefore, $A(S)$ coincides with the positive cone, i.e., one of the two connected components of

$$
\left\{x \in N S(S) \mid\left(x^{2}\right)>0\right\} .
$$

Thus, by replacing $\varphi$ by $-\varphi$ if necessary, both $H_{1}$ and $H_{2}$ are ample on $S$. They are also very ample. In fact, as $N S(S)$ represents neither -2 nor 0 , it follows that $S$ contains neither $\mathbb{P}^{1}$ nor elliptic pencil, and we can apply [SD74] Theorem 5.2. 
From now on, let $S$ be a K3 surface in Lemma 4.4. Corresponding to the complete linear systems $\left|H_{1}\right|,\left|H_{2}\right|$, we can embed $S$ into $\mathbb{P}^{3}$ in two different ways:

$$
\Phi_{\left|H_{1}\right|}: S \simeq S_{1} \subset \mathbb{P}^{3}, \Phi_{\left|H_{2}\right|}: S \simeq S_{2} \subset \mathbb{P}^{3} .
$$

The surfaces $S_{1}$ and $S_{2}$ are quartic surfaces in $\mathbb{P}^{3}$ with no line. Thus, by applying Lemma 4.3 to $S_{1}$ and $S_{2}$ respectively, we have two biholomorphic involutions $\iota_{1}$ and $\iota_{2}$ on $S^{[2]}$. We also note that $N S\left(S^{[2]}\right)=\mathbb{Z}\left\langle H_{1}, H_{2}, e\right\rangle$.

4.5. Lemma. Under the basis $\left\langle H_{1}, e, H_{2}\right\rangle$ of $N S\left(S^{[2]}\right)$, the involutions $\iota_{1}^{*} \mid N S\left(S^{[2]}\right)$ and $\iota_{2}^{*} \mid N S\left(S^{[2]}\right)$ are represented by the following matrices:

$$
M_{1}:=\left(\begin{array}{ccc}
3 & 2 & 8 \\
-4 & -3 & -8 \\
0 & 0 & -1
\end{array}\right), M_{2}:=\left(\begin{array}{ccc}
-1 & 0 & 0 \\
-8 & -3 & -4 \\
8 & 2 & 3
\end{array}\right) .
$$

Proof. By Lemma 4.3, we see that

$$
\iota_{1}^{*}\left(H_{1}\right)=3 H-4 e, \iota_{1}^{*}(e)=2 H_{1}-3 e .
$$

Let us determine $\iota_{1}^{*}\left(H_{2}\right)$. As $N S\left(S^{[2]}\right)=\mathbb{Z}\left\langle H_{1}, e, H_{2}\right\rangle$, one can write

$$
\iota_{1}^{*} H_{2}=a H_{1}+b e+c H_{2} .
$$

Since $\iota_{1}^{*}$ is an isometry, we have

$$
\begin{gathered}
\left(\left(a H_{1}+b e+c H_{2}\right)^{2}\right)=\left(H_{2}^{2}\right)=4, \\
\left(3 H_{1}-4 e, a H_{1}+b e+c H_{2}\right)=\left(H_{1}, H_{2}\right)=8, \\
\left(2 H_{1}-3 e, a H_{1}+b e+c H_{2}\right)=\left(e, H_{2}\right)=0 .
\end{gathered}
$$

From these three equalities, we obtain that

$$
(a, b, c)=(8,-8,-1) \text { or }(4,-8,1) .
$$

Let us exclude the second case $(4,-8,1)$. If this happens, then

$$
\iota_{1}^{*}\left(H_{2}\right)+H_{2}=4 H_{1}-8 e+2 H_{2} .
$$

On the other hand, the class $\iota_{1}^{*}\left(H_{2}\right)+H_{2}$ is invariant under $\iota_{1}$, a contradiction to Lemma 4.3 (3). Thus,

$$
\iota_{1}^{*} H_{2}=8 H_{1}-8 e-H_{2} .
$$

Hence the matrix representation of $\iota_{1}^{*} \mid N S\left(S^{[2]}\right)$ is $M_{1}$ as claimed. Changing the roles of $H_{1}$ and $H_{2}$, we see that the matrix representation of $\iota_{2}^{*} \mid N S\left(S^{[2]}\right)$ is $M_{2}$ as well.

4.6. Lemma. Let $\ell \in[1, \infty) \cap \mathbb{Z}$ and $g_{\ell}=\left(\iota_{2} \iota_{1}\right)^{\ell} \in$ Aut $S^{[2]}$. Then, $g_{\ell}$ is not induced from any Aut $S^{\prime}$. Here $S^{\prime}$ is any K3 surface such that $\left(S^{\prime}\right)^{[2]} \simeq S^{[2]}$. Moreover, we have:

$$
d_{1}\left(g_{\ell}\right)=d_{3}\left(g_{\ell}\right)=(17+12 \sqrt{2})^{\ell}, d_{2}\left(g_{\ell}\right)=(17+12 \sqrt{2})^{2 \ell} .
$$

Proof. By Lemma 4.5, the matrix representation of $\left(\iota_{2} \iota_{1}\right)^{*} \mid N S\left(S^{[2]}\right)$ is

$$
M_{1} M_{2}=\left(\begin{array}{ccc}
45 & 10 & 16 \\
-36 & -7 & -12 \\
-8 & -2 & -3 \\
8 & &
\end{array}\right)
$$


under the basis $\left\langle H_{1}, e, H_{2}\right\rangle$ of $N S\left(S^{[2]}\right)$. By straightforward calculation, we see that the characteristic polynomial of $M_{1} M_{2}$ is

$$
\Phi(x)=(x-1)(x-(17+12 \sqrt{2}))(x-(17-12 \sqrt{2})) .
$$

This together with Theorem 1.1 implies the assertion of dynamical degrees. Here we used the fact that

$$
\rho\left(g_{\ell}^{*} \mid N S\left(S^{[2]}\right)\right)=\rho\left(g_{\ell}^{*} \mid H^{1,1}\left(S^{[2]}, \mathbb{R}\right)\right) .
$$

This equality follows from the fact that the Beauville-Bogolomov-Fujiki's form on the orthogonal complement of $N S(S)_{\mathbb{R}}$ in $H^{1,1}\left(S^{[2]}, \mathbb{R}\right)$ is negative definite.

Let us show that $g_{\ell}$ is not induced from any automorphism of $S^{\prime}$. Suppose that $g_{\ell}$ is induced from some automorphism of $S^{\prime}$. Then, $g_{\ell}^{*}\left(e^{\prime}\right)=e^{\prime}$. Here $E^{\prime}$ is the exceptional divisor of the Hilbert-Chow morphism $\left(S^{\prime}\right)^{[2]} \longrightarrow\left(S^{\prime}\right)^{(2)}$ and $e^{\prime}=E^{\prime} / 2$. On the other hand, by the explicit form of $M_{1} M_{2}$ above, the eigen vector in $N S\left(S^{[2]}\right)$ corresponding to the eigenvalue 1 is $k\left(H_{1}-6 e+H_{2}\right)(k \in \mathbb{Z} \backslash\{0\})$. Thus $e^{\prime}=k\left(H_{1}-6 e+H_{2}\right)$ for some $k \in \mathbb{Z}$. However, this is impossible, as

$$
\left(\left(e^{\prime}\right)^{2}\right)=-2 \text { but }\left(\left(H_{1}-6 e+H_{2}\right)^{2}\right)=-48 \text {. }
$$

Acknowledgement. I would like to express my thanks to Professors Nessim Sibony and Tien-Cuong Dinh for inviting me to Paris (June 2008) and for fruitful discussions. I would like to express my thanks to Professors Frédéric Campana, Kieran G. O'Grady and the refreee for important comments.

\section{REFERENCES}

[Am07] Amerik, E. : A computation of invariants of a rational self-map, arXiv:0707.3947.

[Be82] Beauville, A. : Some remarks on Kähler manifolds with $c_{1}=0$, In: Classification of algebraic and analytic manifolds (Katata, 1982), Progr. Math. 39 Birkhäuser Boston, Boston, MA (1983) 1-26.

[Be83] Beauville, A. : Variétés kälériennes dont la première classe de Chern est nulle, J. Differential Geometry 18 (1983) 755-782.

[BD85] Beauville, A., R. Donagi, La variétés des droites d'une hypersurface cubique de dimension 4, C.R. Acad. Sci. Paris Sér. I Math. bf 301 (1985) 703-706.

[Bo78] Bogomolov, F. A. : Hamiltonian Kählerian manifolds, Dokl. Akad. Nauk SSSR 243 (1978), 1101-1104

[Bo96] Bogomolov, F. A. : On the cohomology ring of a simple hyper-Kähler manifold (on the results of Verbitsky), Geom. Funct. Anal. 6 (1996) 612-618.

[DS05] Dinh, T.-C., Sibony, N. : Green currents for holomorphic automorphisms of compact Kähler manifolds, J. Amer. Math. Soc. 18 (2005) 291-312.

[DS09] Dinh, T.-C., Sibony, N. : Super-potentials for currents on compact Kähler manifolds and dynamics of automorphisms, to appear in J. Algebraic Geometry.

[Fu83] Fujiki, A. : On primitively symplectic compact Kähler $V$-manifolds of dimension four, Classification of algebraic and analytic manifolds (Katata, 1982), 71-250, Progr. Math., 39, Birkhäuser Boston, Boston, MA, 1983.

[Gr03] Gromov, M. : On the entropy of holomorphic maps, Enseign. Math. 49 (2003) 217-235.

[CY03] Gross, M., Huybrechts, D., and Joyce, D. : Calabi-Yau manifolds and related geometries, Universitext. Springer-Verlag, Berlin (2003).

[Gu05] Guedj, V. : Ergodic properties of rational mappings with large topological degree, Ann. of Math. 161 (2005) 1589-1607.

[HT01] Hassett, B., Tschinkel, Y. : Rational curves on holomorphic symplectic fourfolds, Geom. Funct. Anal. 11 (2001) 1201-1228.

[Mc02] McMullen, C. T. : Dynamics on K3 surfaces: Salem numbers and Siegel disks, J. Reine Angew. Math. 545 (2002) 201-233. 
[Mc07] McMullen, C. T. : Dynamics on blowups of the projective plane, Publ. Math. Inst. Hautes Études Sci. 105 (2007) 49-89.

[Mo84] Morrison, D. R. : On K3 surfaces with large Picard number, Invent. Math. 75 (1984) $105-121$.

[OGr05] O'Grady, K. G. : Involutions and linear systems on holomorphic symplectic manifolds, Geom. Funct. Anal. 15 (2005) 1223-1274.

[Og03] Oguiso, K. : Local families of K3 surfaces and applications, J. Algebraic Geometry 12 (2003) 405-433.

[Og07] Oguiso, K. : Salem polynomials and birational transformation groups for hyperKähler manifolds, Sugaku 59 (2007) 1-23.

[SD74] Saint-Donat, B. : Projective models of K-3 surfaces, Amer. J. Math. 96 (1974) 602-639.

[Ve96] Verbitsky, M. : Cohomology of compact hyper-Kähler manifolds and its applications, Geom. Funct. Anal. 6 (1996) 601-611.

[Yo01] Yoshioka, K. : Moduli spaces of stable sheaves on abelian surfaces, Math. Ann. 321 (2001) 817-884.

[Zh08] Zhang, D.-Q. : Dynamics of automorphisms on projective complex manifolds, arXiv:0810.4675, to appear in J. Differential Geometry.

Keiji Oguiso

Department of Economics, Keio University, 4-1-1 Hiyoshi Kohoku-ku, Yokohama, 223-8521, Japan

oguiso@hc.cc.keio.ac.jp 